DE

M E D I C I N A

T R O P I C A L

$\mathrm{DE}$

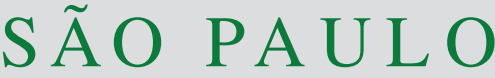

JOURNAL OF THE SÃO PAULO INSTITUTE OF TROPICAL MEDICINE

${ }^{1}$ Universidade Federal do Triângulo Mineiro, Instituto de Ciências Biológicas e Naturais, Uberaba, Minas Gerais, Brazil

2Universidade Federal do Triângulo Mineiro, Serviço de Patologia Cirúrgica, Uberaba, Minas Gerais, Brazil

${ }^{3}$ Universidade Federal de Minas Gerais, Faculdade de Medicina, Laboratório de Pesquisa em Bacteriologia, Belo Horizonte, Minas Gerais, Brazil

${ }^{4}$ Universidade Federal do Triângulo Mineiro, Serviço de Endoscopia, Uberaba, Minas Gerais, Brazil

${ }^{5}$ Universidade Federal do Triângulo Mineiro, Serviço de Gastroenterologia, Uberaba, Minas Gerais, Brazil

Correspondence to: Adriana Gonçalves de Oliveira

Universidade Federal do Triangulo Mineiro, Instituto de Ciências Biológicas e Naturais, Praça Manoel Terra, 330, CEP 38025-180, Uberaba, MG, Brazil

E-mail: adriana.oliveira@uftm.edu.br

Received: 2 October 2018

Accepted: 10 December 2018

\section{Increased serum gastrin in patients with different clinical forms of Chagas disease coinfected with Helicobacter pylori}

\author{
Jacqueline Batista Sousa', Renata Margarida Etchebehere ${ }^{2}$, Dulciene Maria \\ de Magalhães Queiroz ${ }^{3}$, Fernanda Machado Fonseca', Bianca Bontempi \\ Batista $^{1}$, Iracema Saldanha Junqueira4 ${ }^{4}$, Sílvia Maria Perrone Camilo ${ }^{5}$, Adriana \\ Gonçalves de Oliveira ${ }^{(10}$
}

\section{ABSTRACT}

Trypanosoma cruzi and Helicobacter pylori (HP) are pathogens that cause chronic diseases and have been associated with hypergastrinemia. The aim of this study was to evaluate the fasting gastrin levels in patients with different clinical forms of Chagas disease (CD), coinfected or not by HP. The enrolled individuals were outpatients attending at the university hospital. HP infection was assessed by serology and ${ }^{13} \mathrm{C}$-urea breath test. Fasting serum gastrin concentration was measured by chemiluminescence assay. Gastric endoscopic and histological features were also evaluated. Associations between $\mathrm{CD}$ and serum gastrin level were evaluated in a logistical model, adjusting for age, gender and HP status. A total of 113 patients were evaluated (45 with Chagas disease and 68 controls). In the multivariate analysis, increasing serum gastrin levels $(\mathrm{OR}=1.02 ; 95 \% \mathrm{CI}=1.01-1.12)$, increasing age $(\mathrm{OR}=1.05 ; 95 \% \mathrm{CI}=1.02-1.09)$ and HP-positive status $(\mathrm{OR}=2.88 ; 95 \% \mathrm{CI}=1.10$ - 7.51) remained independently associated with $\mathrm{CD}$. The serum gastrin levels were significantly higher in the group of patients with the cardiodigestive form $(P=0.03)$ as well as with digestive form $(P=<0.001)$ of Chagas disease than in the controls. In conclusion, patients with cardiodigestive and digestive clinical forms of $\mathrm{CD}$ have increased basal serum gastrin levels in comparison with controls. Moreover, we also demonstrated that $H$. pylori coinfection contributes to the hypergastrinemia shown in CD.

KEYWORDS: Helicobacter pylori. Chagas disease. Gastrin. Chronic gastritis. Acid secretion. Gastric physiology.

\section{INTRODUCTION}

Chagas disease, caused by the intracellular protozoan Trypanosoma cruzi (T. cruzi), is endemic in Latin American countries where it is primarily transmitted to humans by contact with faeces of triatomine vectors ${ }^{1}$. In the past decades, the migration of populations from endemic areas has contributed to the spread of Chagas disease to the USA, Canada, many European and some Western Pacific countries ${ }^{2}$.

The acute phase of Chagas disease is usually asymptomatic, although a high number of parasites circulate in the bloodstream of infected individuals. Then, the disease progresses to an asymptomatic chronic phase called the indeterminate form, which is prolonged and a few or no parasites are found in blood. Commonly, around $20 \%$ to $30 \%$ of infected patients will develop irreversible cardiovascular and/or gastrointestinal lesions with damage on enteric nervous system. These alterations characterize the symptomatic forms of chronic Chagas disease, i.e., cardiac, digestive or cardiodigestive form ${ }^{1,3}$. 
An abnormally high fasting serum gastrin level associated with hyposecretion of gastric acid has been reported in chagasic patients with the digestive form ${ }^{4-8}$. Gastrin, a hormone produced in $\mathrm{G}$ cells mainly located in the gastric antral mucosa, is a potent secretor of gastric acid. Histamine and acetylcholine, released from enterochromaffin-like cells and from enteric neurons, respectively, also stimulate the acid secretion while somatostatin, secreted by oxyntic and antral $\mathrm{D}$ cells, is the major inhibitor of acid secretion ${ }^{9}$. Indeed, the regulation of gastric acid secretion in parietal cells is achieved by a highly coordinated interaction among neural, hormonal and paracrine pathways.

Gastrin may be increased in other various clinical conditions such as the gastric infection with Helicobacter pylori $(\text { H. pylori })^{10}$. This Gram-negative bacterium is recognized as the main cause of chronic gastritis throughout the world and develops an important role in peptic ulcer, gastric carcinoma and mucosa-associated lymphoid tissue (MALT) lymphoma ${ }^{11}$. H. pylori (HP) causes diverse effects on gastric acid secretion depending mainly on the location within the stomach and the degree of inflammation. Usually, antral predominant gastritis results in hypersecretion of acid and can lead to duodenal ulceration. The predominant gastritis on corpus or pangastritis results in atrophic gastritis and abnormally low secretion of gastric acid associated with marked hypergastrinemia. These alterations can strongly favor the development of gastric adenocarcinoma ${ }^{10,12-14}$.

Studies showing an increased basal serum gastrin levels in patients with Chagas disease evaluated only the digestive form of the disease and most of them were conducted before the discovery of $\mathrm{H}$. pylori, which has been shown to be highly prevalent in chagasic patients. Thus, the aim of this study was to evaluate whether fasting hypergastrinemia also occurs in patients with other clinical forms of Chagas disease, coinfected or not with $H$. pylori.

\section{MATERIAL AND METHODS}

This cross-sectional study was approved by the Ethics Committee of the Federal University of Triangulo Mineiro and was conducted in accordance with the Declaration of Helsinki. All volunteers signed the informed consent form and answered a questionnaire to obtain the socialdemographic data. Clinical information was obtained by reviewing the patients' medical records.

\section{Subjects and clinical samples}

A total of 113 outpatients (45 with Chagas disease and 68 without Chagas Disease (CD) - controls) attended at the university hospital from April 2012 to January 2015 were evaluated. The exclusion criteria were previous therapy for $H$. pylori eradication, history of peptic ulcer, gastrointestinal cancer, renal failure or concomitant severe illness. Individuals taking proton pump inhibitors, $\mathrm{H}_{2}$ blockers and $\mathrm{H}_{2}$-antihistamines or those who underwent upper gastrointestinal tract surgery were also excluded. A blood sample was collected from each patient under fasting conditions for the gastrin measurements, serological diagnosis of Chagas disease and $\mathrm{H}$. pylori infection. Additionally, all patients were submitted to ${ }^{13} \mathrm{C}$-urea breath test $\left({ }^{13} \mathrm{C}\right.$-UBT $)$ for $\mathrm{H}$. pylori diagnostic.

\section{Diagnosis of Chagas disease}

Enzyme-linked immunosorbent assay (ELISA; Chagatest ELISA-WIENER, Rosario, Argentina), passive hemagglutination (Chagatest HAI-WIENER, Rosario, Argentina) and indirect immunofluorescence tests (Imuno-Con Chagas-WAMA, Sao Carlos, Brazil) were performed according to the manufacturer's instructions. Chagasic patients showed positive reactions to at least two serological tests and control patients were negative by the three tests. Chagasic patients were classified according to the clinical form of disease (i.e., indeterminate, digestive, cardiac or cardiodigestive) based on clinical examination, electrocardiography and radiological exams of the chest, esophagus and colon.

\section{H. pylori status}

H. pylori infection was assessed by serology and ${ }^{13} \mathrm{C}$-UBT. Patients with discordant results were excluded. Patients were considered H. pylori-positive (HP-positive) if both tests were positive and $H$. pylori-negative (HP-negative) if both tests were negative. IgG antibodies specific to H. pylori were detected using a commercial ELISA kit (Serion Immundiagnostica GmbH, Würzburg, Germany), according to the manufacturer's instructions and results were expressed as positive or negative. The ${ }^{13} \mathrm{C}-\mathrm{UBT}$ was performed after an overnight fast. After collection of a basal breath sample, an oral dose of ${ }^{13} \mathrm{C}$-urea on a citric acid base was administered. An additional breath sample was collected after $30 \mathrm{~min}$. Breath samples were analyzed using an isotope-selective non-dispersive infra-red spectrometer (NDIRIS; Wagner Analytical Systems, Bremen, Germany). A change $>4.0 \%$ in delta over baseline (DOB) was considered HP-positive.

\section{Measurements of serum gastrin levels}

Gastrin-17 concentration was measured by a chemi- 
luminescence assay (IMMULITE® 2000 immunoassay system; Siemens Medical Solutions Diagnostics, Los Angeles, EUA) and the values were expressed in $\mathrm{pg} / \mathrm{mL}$. All gastrin assays were performed in duplicate. The normal value for fasting gastrin-17 using this assay is $<115 \mathrm{pg} / \mathrm{mL}$.

\section{Histological analysis}

Endoscopic biopsy specimens were obtained from the antrum and corpus of the stomach of patients who underwent EGD by medical indication. Biopsy samples were fixed overnight in buffered formalin embedded in paraffin, sliced to $4 \mu \mathrm{m}$ thickness, and stained with haematoxylin and eosin for histological examination. The Warthin-Starry staining was used for detection of spiral H. pylori. The degree of gastric mucosal inflammation and activity were also assessed. The location of gastritis was classified according to the presence of mononuclear cells in the antrum, corpus or in both. Glandular atrophy and intestinal metaplasia were classified as absent or present.

\section{Statistical analysis}

The association of Chagas disease with the basal serum gastrin level (dependent variable) including $H$. pylori status, age and gender (independent variables) was tested by a univariate analysis. All variables with $P$ values $\leq 0.25$ were included in the full model of the logistic regression. Odds ratios (OR) and 95\% confidence intervals (CI) were used as an estimate of the risk. The Hosmer-Lemeshow goodnessof-fit test was used to evaluate the fitness of the model ${ }^{15}$. In addition to visual examination of histograms and box plots, the Kolmogorov-Smirnov (K-S) test or Shapiro-Wilk (SW) test, based on the number of each subgroups, was used for assessing the normality of data. Because data did not show a normal distribution even after log transformation, a two-tailed Mann-Whitney U-test (median / range) was used to detect the difference of serum gastrin levels among groups. Otherwise, because the age of participants was normally distributed, the groups were compared by a two-tailed Student's T test (mean / standard deviation). Data were analyzed with the statistical package for the social sciences (SPSS) version 23.0 (SPSS Inc., Chicago, IL, USA). For comparison of groups with dichotomous variables the two-tailed chi-squared test (with Yates' correction) or the Fisher's exact test was used. $P$ value $\leq 0.05$ was considered significant.

\section{RESULTS}

\section{Demographic data}

Among the 113 included subjects, 45 were chagasic patients (14 men and 31 women; mean age $64.3 \pm 10.5$ years) and 68 were controls without $\mathrm{CD}(29$ male and 39 female; mean age $60.4 \pm 10.1$ years). When the groups were compared, no difference was observed regarding the gender $(P=0.29 ; \mathrm{OR}=1.65 ; 95 \% \mathrm{CI}=0.74-3.64)$, but the disease group participants were older than the control ones $(P=0.05)$. Most chagasic $(35-77.8 \%)$ patients and controls (43 - 63.2\%) were HP-positive.

\section{Median gastrin levels}

Median serum gastrin levels were higher in chagasic patients than in controls, both in HP-positive patients $(65.10 \mathrm{pg} / \mathrm{mL}$; range, $11-557 \mathrm{pg} / \mathrm{mL}$ vs. $39.80 \mathrm{pg} / \mathrm{mL}$; range, $12-172 \mathrm{pg} / \mathrm{mL}$, respectively; $P=0.01$ ) and HP-negative ones ( $49.15 \mathrm{pg} / \mathrm{mL}$; range, $16-670 \mathrm{pg} / \mathrm{mL}$ vs. $23.0 \mathrm{pg} / \mathrm{mL}$; range, $10-133 \mathrm{pg} / \mathrm{mL}$, respectively; $P=0.009$ ), but higher in the HP-positive groups than in the negative ones.

In order to identify variables independently associated with Chagas disease, data were analyzed in a logistic regression model. In the univariate analysis, the basal serum gastrin level, HP status and age, but not the gender, were selected. In the multivariate analysis, the increasing of serum gastrin levels, the increasing age and HP-positive status remained independently associated with Chagas disease (Table 1).

Table 1 - Variables associated with Chagas disease $(n=45)$ in comparison with controls $(n=68)$ in a multivariate analysis.

\begin{tabular}{lccccc}
\hline \multirow{2}{*}{ Variables } & Univariate & & \multicolumn{3}{c}{ Multivariate } \\
\cline { 2 - 2 } \cline { 5 - 6 } & $P$ value & & OR & $95 \% \mathrm{Cl}$ & $P$ value \\
\hline Gender & 0.29 & & & & \\
Increasing age & 0.05 & & 1.05 & $1.02-1.09$ & 0.04 \\
H. pylori + status & 0.09 & & 2.88 & $1.10-7.51$ & 0.03 \\
Increasing gastrin level & 0.008 & & 1.02 & $1.01-1.12$ & 0.03 \\
\hline
\end{tabular}

The Hosmer-Lemeshow test showed good fitness of the logistic regression model ( $P=0.25 ; 8$ degrees of freedom; 10 steps). + positive; OR- odds ratio; $95 \% \mathrm{Cl}-95 \%$ confidence interval. 
Among chagasic patients, $10(22.2 \%)$ had the cardiodigestive form, $12(26.7 \%)$ the digestive form, $12(26.7 \%)$ the indeterminate form, and $11(24.4 \%)$ the cardiac form of the disease. Figure 1 shows the basal serum gastrin levels of controls and of patients with different clinical forms of Chagas disease. Serum gastrin levels were significantly higher in the group of patients with the cardiodigestive form as well as with the digestive form of disease than in the control group.

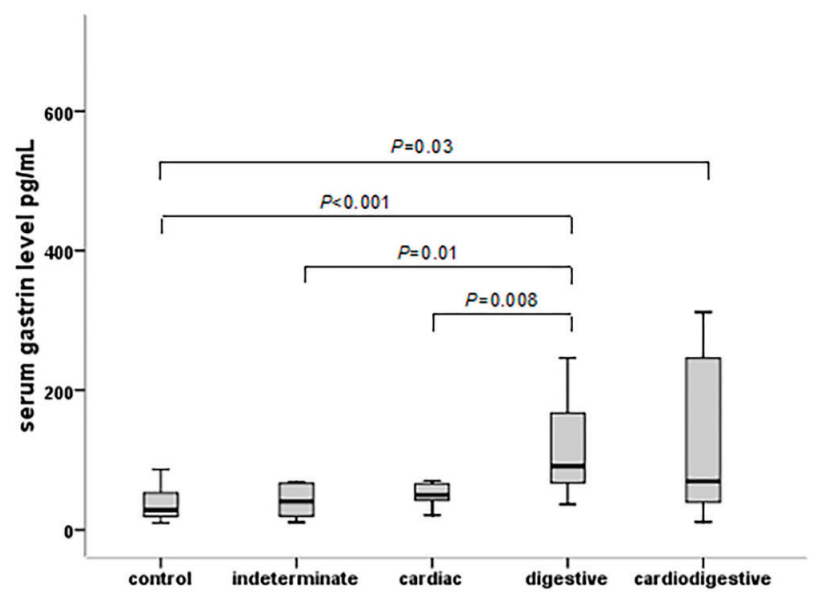

Figure 1 - Box plots representing the basal serum gastrin levels among the control and chagasic patients with different clinical forms of the Chagas disease. The horizontal bar across the box indicates the median and the capped bars indicate the minimum and maximum data values. The upper and lower limits of the boxes represent the $75^{\text {th }}$ and $25^{\text {th }}$ percentiles, respectively. No difference was observed between the controls and the indeterminate $(P=0.53)$ and cardiac $(P=0.13)$ forms of the disease. Also, the cardiodigestive clinical form did not differ from the digestive form $(P=0.65)$. The data were analyzed by the two-tailed Mann-Whitney $U$ test.

Nevertheless, no difference was observed between controls and patients with either the indeterminate $(P=0.53)$ or the cardiac $(P=0.13)$ forms of disease. Also, in the group of chagasic patients, significant higher levels of serum gastrin were observed in the digestive than in the cardiac $(P=0.008)$ and in the indeterminate $(P=0.01)$ forms of the disease. In addition, the cardiodigestive form of the disease did not differ from the digestive one $(P=0.65)$. No significant difference was observed in respect to the prevalence of HP and age among different subgroups of clinical forms of Chagas disease $(P>0.05)$.

In the subgroup of patients who underwent EGD $(\mathrm{n}=35)$, serum gastrin levels were significantly higher $(P=0.004)$ in the nine patients with the digestive clinical form of Chagas disease (median, $72.20 \mathrm{pg} / \mathrm{mL}$; range, 36-246 $\mathrm{pg} / \mathrm{mL}$ ) than in the 26 controls (median, $32.20 \mathrm{pg} / \mathrm{mL}$; range, $11-133 \mathrm{pg} / \mathrm{mL}$ ). Histological exams detected the presence of the bacterium in both, the antral and the oxyntic gastric mucosa in seven $(77.8 \%)$ chagasic patients and in $13(50.0 \%)$ controls $(P=0.29 ; \mathrm{OR}=3.50 ; 95 \% \mathrm{CI}=0.61$ $-20.13)$. Gastritis was observed in the gastric mucosa of all HP-positive patients, without differences in the number of either mononuclear or polymorphonuclear cells between chagasic and control patients ( $P=1.0$ for both, two tailed Fisher exact test). Chagasic patients $(n=4 ; 44.4 \%)$ did not differ from controls $(n=5 ; 19.2 \%)$ in respected to the presence of intestinal metaplasia in the stomach mucosa $(P=0.58 ; \mathrm{OR}=2.17 ; 95 \% \mathrm{CI}=0.45-10.49)$. Gastric or duodenal peptic ulcer was not observed in chagasic patients.

\section{DISCUSSION}

The findings of the present study showed that basal serum gastrin levels are higher in patients with Chagas disease than in non-chagasic controls. This result persisted even after adjustment for potential confounding factors such as age, gender and HP infection. It should be noted that, for the first time, it was demonstrated that hypergastrinemia is present in the cardiodigestive clinical form of Chagas disease as well as in digestive form.

It is known that rising intraluminal $\mathrm{pH}$ of the stomach is the main factor stimulating gastrin production and secretion. However, the abnormal high gastrin levels found in chagasic patients with the digestive clinical form has been attributed to the low gastric acid secretion caused by a partial denervation of the stomach, thereby affecting the parasympathetic stimulation ${ }^{4-8}$. The role of vagal nerves in the stimulation of gastric acid secretion in humans is complex, but it is known that acetylcholine released from these nerves has a direct stimulatory effect on parietal cells ${ }^{14}$. It has been demonstrated that acid hyposecretion and stomach nervous tissue injury are present mainly in the digestive form of Chagas disease. Both conditions can also occur in the cardiodigestive form, as patients presenting with either of these clinical forms of Chagas disease showed significant higher levels of gastrin in comparison with controls. Notably, the cardiodigestive form showed wide values of basal serum gastrin, but without significant difference with the digestive form.

Other mechanisms could also be involved in the increase of serum gastrin levels observed among chagasic patients such as reduced somatostatin secretion, because the significant decrease in the number of antral D-cells observed in CD patients could lead to hypergastrinemia in these patients. Likewise, patients infected with $H$. pylori have abnormal regulation of gastrin secretion due to lower somatostatin concentrations ${ }^{16-19}$ in the antral region of the stomach. Moreover, the eradication of $H$. pylori results in the resolution of the gastritis and associated abnormalities ${ }^{17,18,20}$. 
A high prevalence of coinfection with $H$. pylori was observed among patients with Chagas disease in this study (approximately 78\%) and in others, irrespective of the clinical form of the disease ${ }^{21-26}$. In the histological evaluation, we observed that $H$. pylori infection was spread into the antral and corpus of gastric mucosa in the majority of patients with the digestive form of Chagas disease, which may also contribute to gastric atrophy, hypochlorydria and hypergastrinemia ${ }^{26}$. Thus, the hypergastrinemia that occurs in $\mathrm{H}$. pylori-infected individuals has been attributed to hypochlorhydria, decreased secretion of somatostatin associated with the production of pro-inflammatory cytokines such as IL8, IL1 $\beta$ and TNF $\alpha$, and also by a putative direct induction of the gastrin gene expression by the bacterium itself, especially the more virulent strains $\mathbf{s}^{10,13,27}$.

Taking into account all these considerations, H. pylori gastric infection can contribute to the hypergastrinemia found in Chagas disease. In the present study, we demonstrated, using a logistic regression analysis, that H. pylori infection was not a confounding factor in the increased gastrin levels results observed in Chagas disease.

The gastric physiological changes that could be caused by a long term coinfection with $H$. pylori and $T$. cruzi are completely unknown, but various effects and individual variations can be expected. Therefore, further studies are necessary to clarify the real contribution of the bacterium in the elevated gastrin levels, in chronic Chagas disease. The treatment of chagasic patients coinfected with $H$. pylori and the measurement of their basal gastrin levels after the eradication of the bacterium could bring some highlights on this matter. Among non-chagasic patients, it has been demonstrated that the level of gastrin decreases after H. pylori eradication therapy ${ }^{17,18,20,28}$.

A better understanding of the real influence of $H$. pylori infection on gastrin levels in Chagas disease should lead to an improved management of coinfected patients. Furthermore, as chagasic patients usually have various dyspeptic symptoms and are $H$. pylori coinfected, the diagnosis of $H$. pylori infection and the corresponding eradication therapy should be considered in order to reduce the gastrointestinal symptoms and probably gastrin levels. Pinazzo et al. ${ }^{24}$ reported the resolution or improvement of upper gastrointestinal symptoms in more than $70 \%$ of chagasic patients treated for $H$. pylori infection resulting in a clearer delineation of symptoms directly induced by T. cruzi infection.

The findings of the present study showed that basal serum gastrin levels are higher in patients with Chagas disease than in the non-chagasic ones (controls) even after adjustment for potential confounding factors such as age, gender and HP infection. Additionally, it was demonstrated, for the first time, that hypergastrinemia is present in the cardiodigestive clinical form of Chagas disease as well as in the digestive form.

\section{CONFLICT OF INTERESTS}

The authors declare no conflict of interests.

\section{FUNDING}

This work was supported by the Fundação de Amparo à Pesquisa do Estado de Minas Gerais (FAPEMIG), Brazil.

\section{ACKNOWLEDGMENTS}

We thank Dr. Rodrigo Cunha de Sousa for his assistance with the analysis of patients' clinical data.

\section{AUTHORS' CONTRIBUTIONS}

Jacqueline Batista Sousa participated in the anti-H. pylori IgG detection, collection of demographic data and clinical patient interviews and contributed to the writing of the manuscript; Renata Margarida Etchebehere, is an experienced pathologist who analyzed samples from the gastric mucosa; Dulciene Maria de Magalhães Queiroz analyzed data, performed the statistical analyses and made critical revisions; Fernanda Machado Fonseca participated in the collection of demographic data and clinical data from patients through interviews and analysis of medical records; Bianca Bontempi Batista participated in the urea breath test; Iracema Saldanha Junqueira participated in the esophagogastroduodenoscopy procedure; Sílvia Maria Perrone Camilo participated in patients' follow-up; Adriana Gonçalves de Oliveira conceived, designed the study, analyzed data and drafted the manuscript. All authors read and approved the final manuscript.

\section{REFERENCES}

1. World Health Organization. Research priorities for Chagas disease, human African trypanosomiasis and leishmaniasis. Geneva: WHO; 2012.

2. Castillo-Riquelme M. Chagas disease in non-endemic countries. Lancet Glob Health. 2017;5:e379-80.

3. Prata A. Clinical and epidemiological aspects of Chagas Disease. Lancet Infect Dis. 2001;1:92-100.

4. Padovan W, Godoy RA, Meneghelli UG, Dantas RO, Oliveira $\mathrm{RB}$, Troncon LE. Acid and pepsin secretion in chronic Chagas' disease patients in response to graded doses of pentagastrin and pentagastrin plus bethanecol. Digestion. 1982;23:48-56. 
5. Troncon LE, Oliveira RB, Meneghelli UG, Dantas RO, Godoy RA. Fasting and food-stimulated plasma gastrin levels in chronic Chagas' disease. Digestion. 1984;29:171-6.

6. Rezende JM. The digestive tract in Chagas' disease. Mem Inst Oswaldo Cruz. 1984;79 Suppl:97-106.

7. Troncon LE, Oliveira RB, Meneghelli UG, Dantas RO, Godoy RA. Plasma gastrin and gastric acid response to insulin hypoglycemia in Chagas' disease. Braz J Med Biol Res. 1985;18:273-8.

8. Rocha JR, Ribeiro U, Cecconello I, Sallum RA, Takeda F, Nasi A, et al. Gastric secretory and hormonal patterns in end-stage chagasic achalasia. Dis Esophagus. 2009;22:606-10.

9. Schubert ML. Functional anatomy and physiology of gastric secretion. Curr Opin Gastroenterol. 2015;31:479-85.

10. Dacha S, Razvi M, Massaad J, Cai Q, Wehbi M. Hypergastrinemia. Gastroenterol Rep (Oxf). 2015;3:201-8.

11. Brown LM. Helicobacter pylori: epidemiology and routes of transmission. Epidemiol Rev. 2000;22:283-97.

12. McColl KE, el-Omar E, Gillen D. Helicobacter pylori gastritis and gastric physiology. Gastroenterol Clin North Am. 2000;29:687703.

13. Watson SA, Grabowska AM, El-Zaatari M, Takhar A. Gastrin active participant or bystander in gastric carcinogenesis? Nat Rev Cancer. 2006;6:936-46.

14. Waldum HL, Kleveland PM, Fossmark R. Upper gastrointestinal physiology and diseases. Scand J Gastroenterol. 2015;50:64956

15. Hosmer DW, Lemeshow S. Applied logistic regression. $2^{\text {nd }}$ ed. New York: Wiley; 2000.

16. Kaneko H, Nakada K, Mitsuma T, Uchida K, Furusawa A, Maeda $\mathrm{Y}$, et al. Helicobacter pylori infection induces a decrease in immunoreactive-somatostatin concentrations of human stomach. Dig Dis Sci.1992;37:409-16.

17. Queiroz DM, Mendes EN, Rocha GA, Moura SB, Resende LM, Barbosa AJ, et al. Effect of Helicobacter pylori eradication on antral gastrin- and somatostatin- immunoreactive cell density and gastrin and somatostatin concentrations. Scand J Gastroenterol. 1993;28:858-64.

18. Queiroz DM, Moura SB, Mendes EN, Rocha GA, Barbosa AJ, Carvalho AS. Effect of Helicobacter pylori eradication on G-cell and D-cell density in children. Lancet. 1994;343:1191-3.
19. Liu Y, Vosmaer GD, Tytgat GN, Xiao SD, Ten Kate FJ. Gastrin (G) cells and somatostatin (D) cells in patients with dyspeptic symptoms: Helicobacter pylori associated and non-associated gastritis. J Clin Pathol. 2005;58:927-31.

20. Ohkusa T, Miwa H, Nomura T, Asaoka D, Kurosawa A, Sakamoto $\mathrm{N}$, et al. Improvement in serum pepsinogens and gastrin in long-term monitoring after eradication of Helicobacter pylori: comparison with $\mathrm{H}$. pylori-negative patients. Aliment Pharmacol Ther. 2004;20 Suppl 1:25-32.

21. Barbosa AJ, Queiroz DM, Nogueira AM, Roquette Reis MJ, Mendes EN, Rocha GA, et al. Chronic gastritis and Helicobacter pylori in digestive form of Chagas' disease. Rev Inst Med Trop Sao Paulo. 1993;35:117-21.

22. Oliveira LC, Buso AG, Siqueira Filho L, Moraes F, Oliveira HA, Oliveira RM, et al. Peptic disease and Helicobacter pylori are highly prevalent in patients with the indeterminate form of Chagas' disease: report of 21 cases. Rev Inst Med Trop São Paulo. 1997;39:209-12.

23. Nascimento RS, Valente SR, Oliveira LC. Seroprevalence of Helicobacter pylori infection in chronic chagasic patients, and in the rural and urban population from Uberlândia, Minas Gerais, Brazil. Rev Inst Med Trop São Paulo. 2002;44:251-4.

24. Pinazo MJ, Ignacio Elizalde J, de Jesús Posada E, Gascón J. Co-infection with two emergent old pathogens: Trypanosoma cruzi and Helicobacter pylori. Enferm Infecc Microbiol Clin. 2010;28:751-2.

25. Fonseca FM, Queiroz DM, Rocha AM, Prata A, Crema E, Rodrigues Junior V, et al. Seroprevalence of Helicobacter pylori infection in chagasic and non-chagasic patients from the same geographical region of Brazil. Rev Soc Bras Med Trop. 2012;45:194-8.

26. Fonseca FM, Etchebehere RM, Queiroz DM, Rocha AM, Junqueira IS, Fonseca DN, et al. Histological and endoscopic features of the stomachs of patients with Chagas disease in the era of Helicobacter pylori. Rev Soc Bras Med Trop. 2014;47:739-46.

27. Suzuki H, Moayyedi P. Helicobacter pylori infection in functional dyspepsia. Nat Rev Gastroenterol Hepatol. 2013;10:168-74.

28. Daugule I, Ruskule A, Moisejevs G, Rudzite D, Jonaitis L, Janciauskas D, et al. Long-term dynamics of gastric biomarkers after eradication of Helicobacter pylori infection. Eur J Gastroenterol Hepatol. 2015;27:501-5. 